\title{
The stability of the Circumnuclear Disk clouds in the Galactic Centre
}

\author{
B. Vollmer ${ }^{1,2}$ and W. J. Duschl ${ }^{2,1}$ \\ 1 Max-Planck-Institut für Radioastronomie, Auf dem Hügel 69, 53121 Bonn, Germany \\ 2 Institut für Theoretische Astrophysik der Universität Heidelberg, Tiergartenstraße 15, 69121 Heidelberg, \\ Germany
}

Received 16 February 2001 / Accepted 23 July 2001

\begin{abstract}
The influence of rotation and magnetic fields on the physical properties of isothermal gas clouds is discussed. The presence of rotation and/or magnetic fields results in an increase of the critical cloud mass with respect to gravitational instability for clouds of a given temperature and external pressure. Rotating clouds have higher densities. Consequently, they are more stable against tidal shear than non-rotating clouds. They can approach the Galactic Centre up to a radius of $\sim 2 \mathrm{pc}$ without being disrupted by the tidal shear due to the gravitational potential. For smaller radii the clouds either collapse or become tidally disrupted. We suggest that this mechanism is responsible for the formation of the inner edge of the Circumnuclear Disk in the Galactic Centre.
\end{abstract}

Key words. ISM: clouds - ISM: evolution - Galaxy: center

\section{Introduction}

The Galactic Centre ${ }^{1}$ is surrounded by a large number of gas and dust clouds. The distribution and the kinematics of these clouds are generally interpreted as a thick disk (Circumnuclear Disk CND). Whether this disk-like structure has an outer edge is still a matter of debate. The CND was studied in molecular lines by Gatley et al. (1986) $\left(\mathrm{H}_{2}\right)$, Serabyn et al. (1986) (CO, CS), Güsten et al. (1987) (HCN), DePoy et al. (1989) $\left(\mathrm{H}_{2}\right)$, Sutton et al. (1990) (CO), Jackson et al. (1993) (HCN), and Marr et al. (1993) (HCN), Coil \& Ho (1999, 2000) $\left(\mathrm{NH}_{3}\right)$, Wright et al. (2001) $(\mathrm{HCN})$. The disk has a sharply defined inner edge at a radius of $\sim 1.7$ pc. Inside this radius the gas density drops by more than one order of magnitude. Güsten et al. (1987) suggested that the density of the clouds is not high enough to resist tidal shear due to the centre's strong gravitational field. Therefore, they concluded that the CND is a very short lived structure $\left(\sim 10^{5} \mathrm{yr}\right)$. On the other hand, Jackson et al. (1993) pointed out that the possibility should not be dismissed that the clouds' density is even high enough to stabilize individual gas clouds against tidal

\footnotetext{
Send offprint requests to: B. Vollmer,

e-mail: bvollmer@mpifr-bonn.mpg.de

${ }^{1}$ We assume $8.5 \mathrm{kpc}$ for the distance to the Galactic Centre.
}

disruption $\left(n \sim 10^{7} \mathrm{~cm}^{-3}\right.$ ). In a previous paper (Vollmer \& Duschl 2001; hereafter VD2001) we constructed an analytic model for a clumped gas and dust disk and applied it to the CND. The clouds were treated as isothermal selfgravitating spheres with a given outer pressure. We succeeded in reproducing the main characteristics of the observed gas clouds (mass, central density, density at the outer boundary). A major shortcoming of this model was that these clouds could not resist tidal shear at distances smaller than $\sim 2.5$ pc from the Galactic Centre. In this work we investigate the influence of rotation on the cloud properties and their stability against tidal shear.

\section{Basic picture}

As in VD2001 we assume that during a short accretion event $\left(\Delta t \sim 10^{6} \mathrm{yr}\right)$ an amount of gas of several $10^{4} M_{\odot}$ is driven into the Galactic Centre region to distances less than $10 \mathrm{pc}$. The infalling gas has not a uniform density distribution and might be turbulent. This clumpy medium is exposed to the ambient UV radiation field due to the population of young O/B stars in the Galactic Centre. Low density regions of the infalling gas are evaporated rapidly while regions of higher density stay molecular and are heated to an equilibrium temperature during less than an orbital period. In this way a clumpy circumnuclear disk 
is formed. We assume that the clouds are gravitationally stable. Three kinds of pressure can counterbalance the selfgravitation of the clouds:

(i) Turbulent pressure: if the turbulent pressure is dominant, we can estimate the turbulent velocity dispersion $\sigma$ with the help of the Virial theorem:

$\sigma^{2} \sim \frac{2 G M_{\mathrm{cl}}}{r_{\mathrm{cl}}}$

where $M_{\mathrm{cl}}$ is the cloud mass and $r_{\mathrm{cl}}$ its radius. With $M_{\mathrm{cl}}=$ $30 M_{\odot}$ and $r_{\mathrm{cl}}=0.05 \mathrm{pc}$, the velocity dispersion is $\sigma \sim$ $2.3 \mathrm{~km} \mathrm{~s}^{-1}$ (note that the observed FWHM is two times $\sigma)$.

(ii) Magnetic pressure: in the case of dominant magnetic pressure, we can estimate an equivalent linewidth $c$ using a local magnetic field strength $B=3 \mathrm{mG}$ (Yusef-Zadeh et al. 1996).

$B^{2} / 8 \pi \simeq c^{2} \rho_{\mathrm{cl}}$,

where $\rho_{\mathrm{cl}}$ is the cloud density. With a cloud density of $\rho_{\mathrm{cl}}=3 \times 10^{-18} \mathrm{~g} \mathrm{~cm}^{-3}$ we obtain an equivalent linewidth of $c \sim 3.3 \mathrm{~km} \mathrm{~s}^{-1}$.

(iii) Thermal pressure: if the thermal pressure dominates, the sound velocity at a temperature of $150 \mathrm{~K}$ is $c_{\mathrm{s}} \sim 0.8 \mathrm{~km} \mathrm{~s}^{-1}$. Thus, the linewidth $c$ of a single selfgravitating cloud is $1 \mathrm{~km} \mathrm{~s}^{-1} \leq c \leq 3 \mathrm{~km} \mathrm{~s}^{-1}$.

The observed linewidths are as large as $\Delta v=$ $50 \mathrm{~km} \mathrm{~s}^{-1}$ (see, e.g., Wright et al. 2001). If the clouds within the CND are gravitationally stable, their intrinsic linewidth is much smaller. A possible explanation of the large observed linewidths is an enhanced turbulent velocity dispersion between the clouds due to infalling gas streamers and/or superposition of clouds with different rotational and turbulent velocities (the turbulent velocity dispersion of the model disk of VD2001 is of the order $\left.\Delta v \sim 15 \mathrm{~km} \mathrm{~s}^{-1}\right)$. The study of a $\operatorname{CS}(3-2)$ data cube observed with the IRAM $30 \mathrm{~m}$ Telescope (Zylka et al. 1999) indicates that the features of linewidths up to $50 \mathrm{~km} \mathrm{~s}^{-1}$ might consist of several clouds with small individual linewidths $\left(\sim 2 \mathrm{kms}^{-1}\right)$ (Zylka, private communication).

In the following we discuss rotating clouds without a magnetic field first. As shown above, the inclusion of a magnetic field increases the equivalent linewidth of the clouds by a factor $\sim 3$. The implications of the inclusion of a magnetic field will be discussed in Sect. 6 .

\section{Cloud rotation}

The equilibrium state of rotating isothermal clouds was studied both analytically (Hayashi et al. 1982; Tohline 1985a,b) and numerically (Stahler 1983a,b); Kiguchi et al. 1987; Narita et al. 1990). These authors showed that the density and the mass of rotating clouds can exceed that of non-rotating clouds considerably. Kiguchi et al. (1987) carried out numerical simulations of rotating isothermal spheres embedded in a tenuous intercloud medium, i.e. the outer boundary of the cloud is determined by the external pressure. Their calculations did not include a magnetic field. They concluded that a rotating cloud is dynamically stable if

(i) $M_{\text {crit }}^{\text {rot }} / M_{\text {crit }}^{\mathrm{BE}}<31$, where $M_{\text {crit }}^{\text {rot }}$ is the critical mass for gravitational instability of the rotating cloud and $M_{\mathrm{crit}}^{\mathrm{BE}}$ is the critical mass for gravitational instability of a nonrotating Bonner-Ebert sphere with the same sound speed (temperature) and the same outer pressure;

(ii) the maximum mean rotation velocity is smaller than $2.7 c_{\mathrm{s}}$, where $c_{\mathrm{s}}$ is the sound velocity;

(iii) $\bar{\rho} / \rho_{\text {ext }}<6$, where $\bar{\rho}$ is the mean density of the cloud and $\rho_{\text {ext }}$ is the external density.

Thus, rotating isothermal clouds, which are gravitationally stable are denser and more massive than nonrotating clouds of the same temperature embedded in a medium of the same pressure. We conclude that cloud rotation alters the solution for the physical characteristics of our model clouds (VD2001) in such a way that more massive and denser clouds result which are more stable against tidal disruption.

\section{Specific angular momentum}

\subsection{Cloud formation}

The spin angular momentum of a cloud in the CND should reflect the angular momentum of the interstellar medium from which the cloud has formed (see e.g. Blitz 1993). Within the central $10 \mathrm{pc}$ the gravitational potential is approximatly spherical. The rotation curve is determined for distances smaller than approximatelly 1 pc by the gravitational potential of the central black hole and for distances greater than this by that of the nuclear star cluster (Genzel et al. 1996). If we consider that a cloud forms when a disklike region becomes gravitationally unstable and collapses, we expect a similar result to that considered analytically by Mestel (1966). Since the rotation curve in the Galactic Centre is approximately constant for distances greater $1 \mathrm{pc}$ and is falling inwards for smaller distances, the rotation of the cloud can be either prograde or retrograde depending on the details of the cloud formation. We thus have to estimate the specific angular momentum of the ISM at the place of cloud formation. For an isolated CND, VD2001 found a very small, mean, radial drift velocity of the clouds $\left(v_{\text {drift }}<0.01 \mathrm{~km} \mathrm{~s}^{-1}\right.$; however, a single cloud can approach the Galactic Centre faster). In this case we can assume that the clouds were formed not far away from the galactic distances where we observe them today $\left(2 \mathrm{pc} \leq R_{\mathrm{G}} \leq 7 \mathrm{pc}\right)$. Mestel (1966) has shown that a cloud can have a specific angular momentum about its masscentre up to $J / M_{\mathrm{cl}}=0.5 R^{2} \Omega$, where $R$ is half the size of the collapsed region and $\Omega$ is the local angular velocity. Let us consider a cloud which forms at a distance greater than 2 pc from the Galactic Centre. With a typical density in the disk $\rho=2 \times 10^{-19} \mathrm{~g} \mathrm{~cm}^{-3}$ and a typical cloud mass of $30 M_{\odot}$ (VD2001), the size of the collapsing region is $R \simeq 0.1 \mathrm{pc}$. The initial value of $\Omega$ depends on the details of the collapse. With $\Omega(2 \mathrm{pc}) \leq 60 \mathrm{~km} \mathrm{~s}^{-1} \mathrm{pc}^{-1}$ we obtain a 
specific spin angular momentum of $J / M \leq 0.3 \mathrm{pc} \mathrm{km} \mathrm{s}^{-1}$. If the CND is not isolated, the clouds could have formed at higher distances from the Galactic Centre $(\sim 10 \mathrm{pc})$. Using $\Omega(10 \mathrm{pc}) \sim 12 \mathrm{~km} \mathrm{~s}^{-1} \mathrm{pc}^{-1}$, gives $J / M \leq 6 \times 10^{-2} \mathrm{pc} \mathrm{km} \mathrm{s}^{-1}$. In addition, partially inelastic off-center collisions between the clouds can lead to changes in the spin angular momentum of the clouds.

\subsection{Stability criteria}

Limits on the specific angular momentum of the clouds $J / M$ are given by the onset of bar formation on the one hand and the onset of core collapse on the other hand. Kiguchi et al. (1987) suggested that bar formation occurs for $\beta_{0}>1 / 3$, where

$\beta_{0}=\frac{25}{12}\left(\frac{4 \pi}{3}\right)^{\frac{1}{3}} \frac{\rho_{\mathrm{ext}}^{\frac{1}{3}} J^{2}}{G M^{\frac{10}{3}}}$

is the ration of rotational energy to gravitational energy ratio of the cloud. Here $\rho_{\text {ext }}$ is the boundary density of the cloud, $J$ is the angular momentum of the cloud, $M$ its mass, and $G$ the gravitation constant. The criterion for clouds which are stable against bar formation is thus

$J / M \leq 0.31 G^{\frac{1}{2}} \rho_{\text {ext }}^{-\frac{1}{6}} M^{\frac{2}{3}} \sim 7 \times 10^{-2} \mathrm{pc} \mathrm{km} \mathrm{s}^{-1}$,

where we have used typical cloud parameters $\left(M=30 M_{\odot}, \rho_{\text {ext }}=3 \times 10^{-19} \mathrm{~g} \mathrm{~cm}^{-3}\right)$. Miyama et al. (1984) found that rotating isothermal spheres collapse if $\alpha_{0} \beta_{0}<0.2$, where

$\alpha_{0}=\frac{5}{2}\left(\frac{4 \pi}{3}\right)^{-\frac{1}{3}} \frac{c_{\mathrm{s}}^{2}}{G \rho_{\mathrm{ext}}^{\frac{1}{3}} M^{\frac{2}{3}}}$

is the thermal to gravitational energy of the cloud. The criterion for stable clouds thus is

$J / M \geq 0.2 \frac{G M}{c_{\mathrm{s}}} \sim 5 \times 10^{-2} \mathrm{pc} \mathrm{km} \mathrm{s}^{-1}$,

using the cloud parameters from above and $c_{\mathrm{s}}=1 \mathrm{kms}^{-1}$.

In Sect. 6 it is shown that the presence of magnetic fields with a field strength of several $\mathrm{mG}$ results in an effective linewidth of $c \sim 3 \mathrm{~km} \mathrm{~s}^{-1}$. In this case the specific angular momentum is

$J / M \geq 0.2 \frac{G M}{c} \sim 2 \times 10^{-2} \mathrm{pc} \mathrm{km} \mathrm{s}^{-1}$.

We thus conclude that for a stable rotating cloud $2 \times 10^{-2} \leq J / M \leq 7 \times 10^{-2} \mathrm{pc} \mathrm{km} \mathrm{s}^{-1}$. This is consistent with the upper limits $6 \times 10^{-2} \leq J / M \leq 0.3 \mathrm{pc} \mathrm{km} \mathrm{s}^{-1}$ at the moment of cloud formation (Sect. 4.1).

\section{A typical rotating cloud}

The maximum central density $\rho_{\mathrm{c}}$ of an isothermal cloud with external pressure $P_{\text {ext }}$ (Bonner-Ebert sphere) is $\rho_{\mathrm{c}} / \rho_{*}=14$, where $\rho_{*}=c_{\mathrm{s}}^{-2} P_{\text {ext }}$. Stable rotating isothermal clouds can have central densities up to $\rho_{\mathrm{c}} / \rho_{*} \sim 100$.
Figure 14 of Kiguchi et al. (1987) shows the region of stable clouds as a function of the central density and the cloud mass. In our case $M_{*}=c_{\mathrm{s}}^{4} P_{\text {ext }}^{-\frac{1}{2}} G^{-\frac{3}{2}}=15 M_{\odot}$ and $\rho_{*}=10^{-18} \mathrm{~g} \mathrm{~cm}^{-3}$. Since we are interested in rotating clouds that have higher central densities than non-rotating clouds, we will only discuss the region for $\rho_{\mathrm{c}} / \rho_{*}>14$. In this case our region of interest is limited by the dashed collapse curve and the dash-dotted curve for bar formation in Fig. 14 of Kiguchi et al. (1987). A typical cloud in this region has $M / M_{*} \sim 2-3$ or $\left(\rho_{\mathrm{c}} / \rho_{*}\right)^{\text {rot }} \sim 3\left(\rho_{\mathrm{c}} / \rho_{*}\right)^{\mathrm{BE}}$. Such a cloud has the following physical characteristics (Kiguchi et al. 1987; Table 3): $\beta_{0}=0.16, \rho_{\mathrm{c}} / \rho_{*}=30, M / M_{*}=$ $2.38, c_{\mathrm{s}} J /\left(G M^{2}\right)=0.164, \bar{\rho} / \rho_{*}=2.71$. In physical units this gives: $M=36 M_{\odot}, \bar{\rho}=2.7 \times 10^{-18} \mathrm{~g} \mathrm{~cm}^{-3}$, $\rho_{\mathrm{c}}=3 \times 10^{-17} \mathrm{~g} \mathrm{~cm}^{-3}$. The radius perpendicular to the rotation axis is $r_{\mathrm{e}}=8 \times 10^{-2} \mathrm{pc}$ and parallel to the rotation axis $z=3 \times 10^{-2} \mathrm{pc}$. The maximum mean rotation velocity is $v_{\text {rot }} \sim 0.8 \mathrm{~km} \mathrm{~s}^{-1}$.

This cloud has thus a 3 times higher central density than a non-rotating isothermal selfgravitating sphere of the same temperature which is embedded in a tenuous medium of the same outer pressure.

The clouds are illuminated by the UV radiation field coming from the central He I star cluster. The mass loss rate of these clouds due to outflowing ionized gas is approximately $\dot{M} \sim 4 \pi r_{\mathrm{cl}}^{2} \rho_{\mathrm{cl}} c_{\mathrm{i}}$, where $r_{\mathrm{cl}}$ is the cloud radius and $c_{\mathrm{i}}$ is the sound velocity in the external ionized gas. Since for the diameter of such a cloud $d_{\mathrm{cl}}^{\mathrm{rot}}<2 \times d_{\mathrm{cl}}^{\mathrm{BE}}$, rotation lowers the lifetime of a cloud by a factor smaller than 4 . The minimum lifetime is still greater than $10^{7} \mathrm{yr}$.

\section{Magnetic fields}

Tomisaka et al. (1989) studied the structure of selfgravitationally stable magnetized clouds. They showed that the critical mass for stability is

$M_{\mathrm{mag}} \sim 1.18\left(1-\left(\frac{0.17}{G^{\frac{1}{2}}\left|\mathrm{~d} M_{\mathrm{cl}} / \mathrm{d} \Phi_{\mathrm{B}}\right|_{\mathrm{c}}}\right)^{2}\right)^{-\frac{3}{2}} M_{\mathrm{BE}}$

where $\left|\mathrm{d} M_{\mathrm{cl}} / \mathrm{d} \Phi_{\mathrm{B}}\right|_{\mathrm{c}}$ represents the mass-to-magnetic flux ratio at the center and $M_{\mathrm{BE}}$ is the Bonner-Ebert critical mass in the absence of magnetic fields. If we approximate $\left|\mathrm{d} M_{\mathrm{cl}} / \mathrm{d} \Phi_{\mathrm{B}}\right|_{\mathrm{c}} \simeq M_{\mathrm{cl}}\left(B r_{\mathrm{cl}}^{2} / 2\right)^{-1}$ and let $M_{\mathrm{cl}}=30 M_{\odot}, r_{\mathrm{cl}}=0.05 \mathrm{pc}$, and $B=5 \mathrm{mG}$, we obtain $M_{\text {mag }} \sim 2.5 M_{\mathrm{BE}}$. This is consistent with a realistic Bonner-Ebert mass of $M_{\mathrm{BE}} \sim 10 M_{\odot}$ (VD2001). We would thus expect that the density of a magnetized cloud of size $d_{\mathrm{cl}}=0.1 \mathrm{pc}$, mass $M_{\mathrm{cl}}=30 M_{\odot}$, and magnetic field strength $B$ of several $\mathrm{mG}$ would also show a density enhancement of a factor 3 with respect to a non-rotating, non-magnetized isothermal, selfgravitating cloud. 


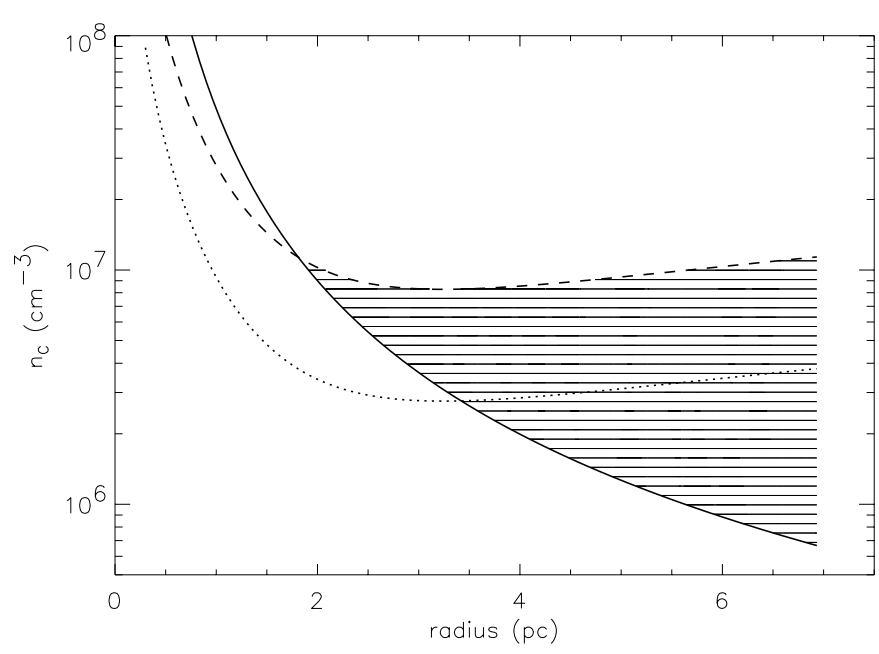

Fig. 1. Central density of the clouds as a function of the distance from the Galactic Centre. Solid: critical density for tidal disruption. Dotted: model central density of VD2001. Dashed line: central density of a rotating cloud. Dashed surface: range of densities where clouds are gravitationally and tidally stable.

\section{Tidal shear}

The critical density for a cloud orbiting at a distance $R$ on a circular orbit around a point mass $M$ below which it will disintegrate due to tidal shear is given by

$\rho_{\text {crit }}=\frac{3}{2 \pi} \frac{M}{R^{3}}$

(see e.g. Stark et al. 1989). We approximate the mass distribution in the Galactic Centre by $M(R)=M_{0}+M_{1} R^{1.25}$, where $M_{0}=3 \times 10^{6} M_{\odot}$ an $M_{1}=1.6 \times 10^{6} M_{\odot} \mathrm{pc}^{-1.25}$ (this is close to the findings of Eckart \& Genzel 1996). The obtained critical density can be compared to the model density of VD2001 (Fig. 1). For these clouds only selfgravitation and thermal pressure of the neutral and ionized gas are taken into account. Since our model clouds are on the edge of gravitational instability, their central density is close to the critical density for gravitational collapse (Fig. 1 dotted line). As long as this central density is higher than the critical central density with respect to tidal shear (Fig. 1 solid line), non-rotating isothermal clouds can exist. The crossing of the curves corresponds thus to the minimum distance where stable clouds can exist. This critical Galactic radius is $R_{\text {crit }} \sim 3.5$ pc for this estimate. More detailed calculations show $R_{\text {crit }} \sim 2.5$ pc for the illuminated side and $R_{\text {crit }} \leq 3.5 \mathrm{pc}$ for the shadowed side. This difference is due to the different mechanisms that create the cloud boundary at the illuminated and shadowed side. At the illuminated side, the density of the ionized gas is given by the ionization-recombination equilibrium. The heated and ionized gas flows away from the cloud and fills the space between the clouds. In this way, a low density ionized interclump medium $\left(n_{\mathrm{e}} \sim 10^{3} \mathrm{~cm}^{-3}\right)$ is built up. The external pressure of this interclump medium is responsible for the outer edge of the cloud at the shadowed side.
We have argued in the previous sections that for a typical rotating or magnetized cloud $\rho_{\mathrm{c}}^{\mathrm{rot} / \mathrm{mag}} \sim 3 \times \rho_{\mathrm{c}}^{\mathrm{BE}}$ (Fig. 1 dashed line). The presence of rotation and/or magnetic fields alters the properties of the cloud, i.e. they are more massive and have higher central densities. The critical Galactic radius thus decreases to $R_{\text {crit }} \sim 2$ pc. In the framework of our model and its approximations, this is in agreement with the observed inner edge of the CND at $R_{\mathrm{G}} \sim 1.7 \mathrm{pc}$. The dependence of the inner edge on the UV radiation field will be discussed in Sect. 9.

\section{The influence of the stellar winds}

The stellar wind emanating from the central HeI star cluster which is responsible for the UV radiation field exerts a ram pressure on the illuminated side of the cloud:

$$
\begin{aligned}
& P_{\mathrm{ram}} \simeq 2.5 \times 10^{-8} \\
& \times \frac{\left(\dot{M} / 3 \times 10^{-3} M_{\odot} \mathrm{yr}^{-1}\right)\left(v / 600 \mathrm{~km} \mathrm{~s}^{-1}\right)}{4 \pi(R / 2 \mathrm{pc})^{2}} \mathrm{erg} \mathrm{cm}^{-3},
\end{aligned}
$$

(Yusef-Zadeh \& Wardle 1993), where $\dot{M}$ is the stellar mass loss rate due to a wind of velocity $v$. The energy density due to the selfgravity of the cloud is given by

$$
\begin{aligned}
P_{\text {grav }} & \simeq 5 \times 10^{-8}\left(n_{\mathrm{cl}} / 10^{6} \mathrm{~cm}^{-3}\right) \\
& \times \frac{\left(M_{\mathrm{cl}} / 30 M_{\odot}\right) G}{\left(r_{\mathrm{cl}} / 0.05 \mathrm{pc}\right)} \mathrm{erg} \mathrm{cm}^{-3},
\end{aligned}
$$

where $M_{\mathrm{cl}}$ and $r_{\mathrm{cl}}$ are the cloud mass and mean radius. Ram pressure is thus able to push the ionized and heated gas $\left(n_{\mathrm{i}} \sim\right.$ several $\left.10^{3} \mathrm{~cm}^{-3}\right)$ radially away from the Galactic Centre, and might even shape the neutral condensations at the inner edge of the CND. It represents an additional external force on the illuminated side of clouds at the inner edge of the CND.

\section{The inner edge of a CND}

If we assume a spectrum of clouds moving around a galactic centre forming a disk-like equilibrium structure, there are four effects that determine the physical properties of the clouds: UV radiation, tidal shear, a radially-directed wind, and selfgravitation. In the following considerations we will neglect the effects of stellar winds. Nevertheless, one has to keep in mind that they can provide an additional external pressure on the clouds at the inner edge of the CND. Only those clouds with a sufficiently high central density can resist tidal disruption. Thus, the clouds' mean density must increase with decreasing distance to the Galactic Centre. If they reach the Jeans mass they become gravitationally unstable and collapse. Taking these two effects together we obtain an efficient mechanism to create an inner edge. At this distance, the clouds that can resist tidal disruption become Jeans unstable, i.e. the dense cloud structure is lost. The UV radiation plays an important rôle in determining the radius of the clouds at each distance from the galactic centre. With an increasing UV radiation field the cloud radius decreases, because 
the ionization front in direction of the central star cluster is located at smaller cloud radii (Dyson 1968). At these radii the enhanced pressure of the ionized gas in the ionization front is counterbalanced by the enhanced thermal pressure due to the increasing density of the neutral gas in the isothermal cloud. Thus the cloud mass decreases with increasing UV radiation field and the cloud is less susceptible to gravitational collapse.

We can estimate this effect quantitatively in the following way. In the case of a cloud with uniform density the maximum density needed to stabilize a cloud against tidal forces is

$\rho_{\text {crit }}^{\text {tidal }}=\frac{3}{2 \pi} \frac{M(R)}{R^{3}}$,

where $M(R)$ is the enclosed mass up to the radius $R$ from the Galactic Centre. The criterion for Jeans instability is given by

$\rho_{\mathrm{crit}}^{\mathrm{Jeans}}=\frac{\pi \mathcal{R} T}{G \mu r_{\mathrm{cl}}^{2}}$

where $T$ is the temperature of the cloud, $\mathcal{R}$ is the gas constant, $G$ is the gravitational constant, and $\mu$ is the molecular weight.

The cloud radius due to the balance of ionization and recombination is given by Dyson (1968)

$r_{\mathrm{cl}}=\xi^{2} J_{0} n_{\mathrm{i}}^{-2}$,

where $J_{0}$ is the number of incident UV photons per $\mathrm{cm}^{2}$ and $\mathrm{s}, n_{\mathrm{i}}$ is the number density of the ionized gas in the ionization front, and $\xi=4.87 \times 10^{6} \mathrm{~cm}^{-\frac{3}{2}} \mathrm{~s}^{\frac{1}{2}}$. With the jump condition across the ionization front $\rho_{\mathrm{cl}} \mathrm{e}^{-u} c_{\mathrm{s}}^{2}=$ $2 \rho_{\mathrm{i}} c_{\mathrm{i}}^{2}$, where $\rho_{\mathrm{i}}$ and $c_{\mathrm{i}}$ are the density and the sound velocity of the external ionized gas and $u(x)$ is a parameter of the Lane-Emden equation, one can write

$r_{\mathrm{cl}}=4 \xi^{2} m_{p}^{2} J_{0} \rho_{\mathrm{cl}}^{-2} \mathrm{e}^{2 u}\left(\frac{c_{\mathrm{i}}}{c_{\mathrm{S}}}\right)^{4}$.

Furthermore, one can approximate the function $\mathrm{e}^{-u(x)} \sim$ $3 x^{-2}$ for $2<x<8$, where $x=r_{\mathrm{cl}} / c_{\mathrm{s}} \sqrt{4 \pi G \rho_{\mathrm{c}}}$. This leads to

$r_{\mathrm{cl}}=3.645 \times 10^{15} J_{0}^{-\frac{1}{3}} c_{\mathrm{i}}^{-\frac{4}{3}} c_{\mathrm{s}}^{\frac{8}{3}}$.

Thus, the cloud radius depends only on the number of incident UV photons, the sound speed of the ionized gas, and the sound speed of the neutral gas. If we assume that the temperature is determined by the radiation field $c_{\mathrm{s}} \propto T^{\frac{1}{2}} \propto J_{0}^{\frac{1}{8}}$, the cloud radius does not change with the cloud's distance to the Galactic Centre. This means that the cloud's radius is constant and does not depend on its central density. Consequently, the critical Jeans density $\rho_{\text {crit }}^{\text {Jeans }}$ is proportional to the gas temperature of the neutral gas.

Figure 2 shows the critical densities with respect to tidal shear and gravitational collapse. These graphs represent an approximation of the density variations calculated from the detailed model of VD2001 (Fig. 1). The increase

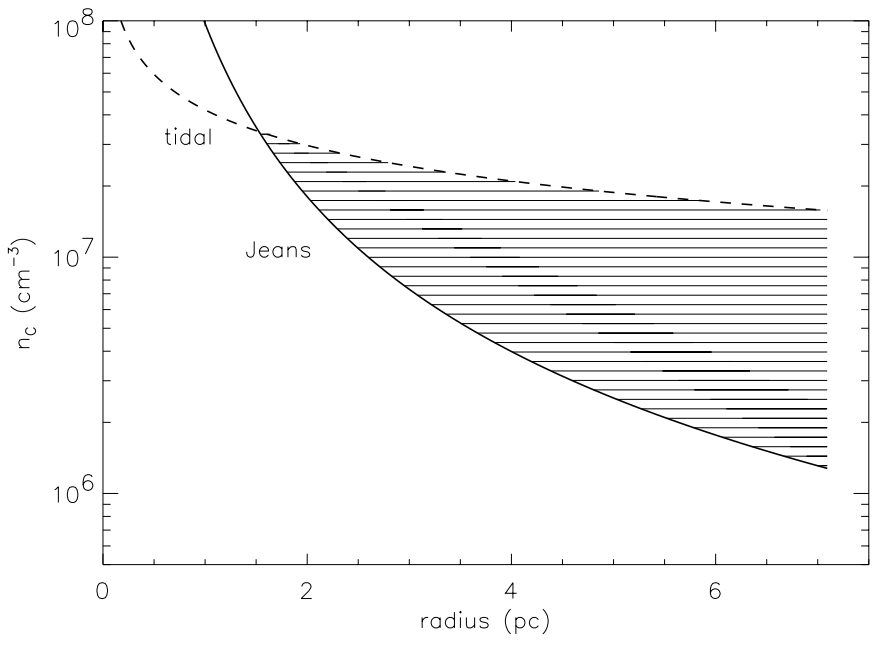

Fig. 2. Central density of the heavy clouds versus the distance to the Galactic Centre. Dashed line: maximum central density above which gravitational collapse occurs. Solid line: minimum density in order to resist tidal shear. Dashed surface: range of densities where clouds are gravitationally and tidally stable.

or decrease of the neutral gas temperature by a factor 2 results in a variation of the location of the inner edge of \pm 1 pc. In Fig. 2 the dashed line shows the maximum central density above which gravitational collapse occurs ( $\rho_{\text {crit }}^{\text {Jeans }}$ using Eq. $\left.(16)\right)$, the solid line shows the minimum density in order to resist tidal shear $\left(\rho_{\text {crit }}^{\text {tidal }}\right)$. The dashed surface shows the range of densities where clouds are gravitationally and tidally stable. The inner edge of the CND arises thus naturally as a selection effect due to external conditions of the environment on the cloud spectrum.

If there are non-negligible non-thermal pressure components, we have to add these components to the sound speed of the neutral and ionized gas. For a non-thermal linewidth $c>1 \mathrm{~km} \mathrm{~s}^{-1}$, the critical Jeans density is always lower than the critical tidal density, i.e. no stable clouds can exist. Therefore, we suggest that only rotating clouds, which have a higher central density can survive.

\subsection{A possible scenario for star formation}

In the previous Sections we have investigated an isolated clumpy gas disk. However, the CND appears to interact with the surrounding gas. Coil \& Ho $(1999,2000)$ and Zylka et al. (1999) conclude on the basis of the gas distribution and kinematics in the inner 20 pc of the Galactic Centre that there are connections between the CND and the neighbouring GMCs. They claim that there are several streamers that fall into the Galactic Centre.

From the theoretical point of view Sanders (1998) pointed out the possibility that the CND can be understood in terms of tidal capture and disruption of gas clouds falling into the Galactic Centre region. The infalling gas forms a tidally stretched filament intersecting itself. After several rotation periods the gas forms a stable ring structure which can be maintained for more than $10^{6} \mathrm{yr}$. He showed that the central star cluster can be created within 
the first few passages of the cloud when the long filament intersects itself at a large angle.

We will now discuss what happens when a cloud complex falls from a distance greater than $10 \mathrm{pc}$ into the Galactic Centre.

In VD2001 we have shown that the CND has a lifetime of $\sim 10^{7} \mathrm{yr}$. It is thus possible that an external cloud is falling into the Galactic Centre within this period. We propose a new scenario in which a whole cloud complex is falling into the Galactic Centre where a clumpy disk structure already exists. When the cloud hits the CND, frequent partially inelastic cloud-cloud collisions will create a whole transient spectrum of clouds with different masses. Those clouds which have masses above the Jeans limit will collapse and eventually form stars. The massive stars are thus formed within a very short time during the collision of the cloud complex and the disk. Partially inelastic collisions result in a decrease of the cloud velocities. Low velocity clouds $\left(v<v_{\text {rot }} \sim 120 \mathrm{~km} \mathrm{~s}^{-1}\right)$ form stars and approach the Galactic Centre at the same time. Thus, if an external gas cloud collides with a pre-existing CND, we expect that a part of the gas, which loses angular momentum due to cloud-cloud collisions spirals inwards with a velocity of $\sim 100 \mathrm{~km} \mathrm{~s}^{-1}$.

If we assume an initial He I star mass of $M_{0}=30 M_{\odot}$ with an initial velocity of $V_{0}=100 \mathrm{~km} \mathrm{~s}^{-1}$, falling into an already existing old stellar cluster of density $\rho_{\text {stellar }}=4 \times 10^{6} M_{\odot} \mathrm{pc}^{-3}$ with stellar masses around $1 M_{\odot}$, collective relaxation dominates over the particleparticle relaxation (Saslaw 1985). This leads to a relaxation time of

$$
\begin{aligned}
\tau_{R, \text { coll }}= & 7 \times 10^{6} \times\left(\frac{V_{0}}{100 \mathrm{~km} \mathrm{~s}^{-1}}\right)^{3} \\
& \times\left(\frac{M_{0}}{30 M_{\odot}}\right)^{-1}\left(\frac{\rho_{\text {stellar }}}{4 \times 10^{6} \frac{M_{\odot}}{\mathrm{pc}^{3}}}\right)^{-1} \mathrm{yr} .
\end{aligned}
$$

A single massive star, whose velocity is approximately the Keplerian velocity of gas moving on circular orbits around the Galactic Centre, thus loses the information about its initial position and velocity within $\sim 7 \times 10^{6} \mathrm{yr}$.

Gerhard (2001) estimated that a star cluster of $\sim 10^{5} M_{\odot}$ which is formed at a Galactic radius $R_{\mathrm{G}}=10 \mathrm{pc}$ needs several Myr to spiral into the Galactic Centre. This timescale is comparable to our collective relaxation timescale.

The star cluster relaxation time is thus comparable to the lifetime of the HeI stars. These massive stars are formed at the same time and at the same distance to the Galactic Centre forming a star cluster after a few million years. The observed streaming motions of the HeI stars in the Galactic Centre (Genzel et al. 1996) shows that the star cluster is not completely relaxed. Therefore, it is possible that the central HeI star cluster has been built during the collision of an infalling cloud complex with an already existing CND.

\section{Conclusion}

We investigated the influence of rotation and magnetic fields on the physical properties of the gas clouds located in the Circumnuclear Disk in the Galactic Centre. Rotating selfgravitating isothermal clouds of a given temperature $T$ embedded in a tenuous medium giving rise to an external pressure $P_{\text {ext }}$ are more massive and have larger densities than non-rotating clouds of same $T$ and $P_{\text {ext }}$. Stable rotating clouds have an angular momentum in the range $3 \times 10^{-2} \leq J / M \leq 5 \times 10^{-2} \mathrm{pc} \mathrm{km} \mathrm{s}^{-1}$. This represents $\sim 20 \%-100 \%$ of the maximum specific angular momentum that a cloud can acquire during its formation. These clouds are stable against tidal shear for Galactic Radii $R_{\mathrm{G}} \geq 2$ pc. Magnetized selfgravitating isothermal clouds with magnetic field strengths of several $\mathrm{mG}$ have a critical mass with respect to gravitational collapse of $\sim 3$ times the corresponding Bonner-Ebert critical mass of a selfgravitating isothermal sphere.

We suggest a mechanism for the formation of an inner edge in circumnuclear disks. The external UV radiation field determines the diameter of the clouds. The density of the clouds must increase with decreasing galactic radius because of the tidal shear due to the gravitational potential in the Galactic Centre. At a critical radius clouds that are stable against tidal shear become gravitational unstable and collapse. On the basis of our model, selfgravitating non-magnetized isothermal clouds should rotate in order to resist tidal shear at a distance of $2 \mathrm{pc}$ from the Galactic Centre, whereas magnetized clouds must rotate in order to resist tidal shear. For the CND in the Galactic Centre the critical radius corresponds approximately to the observed inner edge if cloud rotation is included. We suggest that the central HeI star cluster has been formed when an external cloud collided with a pre-existing CND.

Acknowledgements. We would like to thank P. Ho for helping us to improve this article significantly.

\section{References}

Blitz, L. 1993, in Protostars and Planets III (University of Arizona Press) 125

Coil, A. L., \& Ho, P. T. P. 1999, ApJ, 513, 752

Coil, A. L., \& Ho, P. T. P. 2000, ApJ, 533, 245

DePoy, D. L., Gatley, I., \& McLean, I. S. 1989, IAU Symp., 136,361

Dyson, J. E. 1968, ApSS, 1, 388

Eckart, A., \& Genzel, R. 1996, Nature, 383, 415

Gatley, I., Jones, J. J., Hyland, A. R., et al. 1986, MNRAS, 222, 299

Genzel, R., Thatte, N., Krabbe, A., et al. 1996, ApJ, 472, 153

Gerhard, O. 2001, ApJ, 546, L39

Güsten, R., Genzel, R., Wright, M. C. H., et al. 1987, ApJ, 318,124

Hayashi, C., Narita, S., \& Miyama, S. M. 1982, Prog. Theor. Phys., Osaka, 68, 1949

Jackson, J. M., Geis, N., Genzel, R., et al. 1993, ApJ, 402, 173

Kiguchi, M., Narita, S., Miyama, S. M., \& Hayashi, C. 1987, ApJ, 317, 830 
Marr, J. M., Wright, M. C. H., \& Backer, D. C. 1993, ApJ, 411,667

Mestel, L. 1966, MNRAS, 131, 307

Miyama, S. M., Hayashi, C., \& Narita, S. 1984, ApJ, 279, 621

Narita, S., Kiguchi, M., Miyama, S. M., \& Hayashi, C. 1990, MNRAS, 244, 349

Sanders, R. H. 1998, MNRAS, 1998, 294

Saslaw, W. C. 1985, Gravitational physics of stellar and galactic systems (Cambridge University Press)

Serabyn, E., Güsten, R., Walmsley, C. M., et al. 1986, A\&A, 169,85

Stahler, S. W. 1983a, ApJ, 268, 155

Stahler, S. W. 1983b, ApJ, 268, 165

Stark, A. A., Bally, J., Wilson, R. W., \& Pound, M. W. 1989, IAU Symp., 135, 129
Sutton, E. C., Danchi, W. C., Jaminet, P. A., \& Masson, C. R. 1990, ApJ, 348, 503

Tohline, J. E. 1985a, Icarus, 61, 10

Tohline, J. E. 1985b, ApJ, 292, 181

Tomisaka, K., Ikeuchi, S., \& Nakamura, T. 1989, ApJ, 341, 220

Vollmer, B., \& Duschl, W. J. 2001, A\&A, 367, 72 (=VD2001)

Wright, M. C. H., Coil, A. L., McGary, R. S., Ho P. T. P., \& Harris A. I. 2001, ApJ, 551, 254

Yusef-Zadeh, F., \& Wardle, M. 1993, ApJ, 405, 584

Yusef-Zadeh, F., Roberts, D. A., Goss, W. M., Frail, D. A., \& Green A. J. 1996, ApJ, 466, L25

Zylka, R., Güsten, R., Philipp, S. et al. 1999, in The Central Parsecs of the Galaxy, ed. H. Falke, A. Cotera, W. J. Duschl, F. Melia, \& M. J. Rieke, ASP Conf. Ser., 186, 415 